\title{
Outcomes of 23G vitrectomy and internal limiting membrane peeling with brilliant blue in patients with myopic foveoschisis from a retrospective cohort study
}

\author{
XINBANG MAO, ZHIPENG YOU and YANHUA CHENG
}

Department of Ophthalmology, The Second Affiliated Hospital of Nanchang University, Nanchang, Jiangxi 330006, P.R. China

Received June 14, 2018; Accepted February 21, 2019

DOI: $10.3892 / \mathrm{etm} .2019 .7610$

\begin{abstract}
The aim of the present study was to investigate whether internal limiting membrane (ILM) peeling in patients with myopic foveoschisis (MF) treated with 23-gauge (23G) vitrectomy improved the anatomical and visual outcomes. In this retrospective cohort study, from March 2014 to August 2017 at the Department of Ophthalmology, The Second Affiliated Hospital of Nanchang University (Nanchang, China), 60 patients (60 eyes) with MF underwent $23 \mathrm{G}$ vitrectomy. The patients were grouped according to whether they underwent brilliant blue-assisted ILM peeling (peeling group) or not (non-peeling group). Best-corrected visual acuity (BCVA) and central macular thickness (CMT) on optical coherence tomography were measured. There were 30 eyes in each group and the baseline characteristics were similar (all $\mathrm{P}>0.05$ ). BCVA improved in 24 eyes (80\%) in the peeling group and 25 eyes $(83.3 \%)$ in the non-peeling group $(\mathrm{P}=0.922)$. Preoperative CMT was not significantly different between the peeling and non-peeling group $(458 \pm 62.2$ vs. $460 \pm 61.1 \mu \mathrm{m}$, respectively, $\mathrm{P}=0.229$ ). However, postoperative $\mathrm{CMT}$ was significantly different between the peeling and non-peeling group (269.3 \pm 67.7 vs. $294.4 \pm 60.5 \mu \mathrm{m}$, respectively; $\mathrm{P}=0.015)$. In the peeling group, MF was completely resolved in all 30 eyes, but only in 26 eyes in the non-peeling group $(\mathrm{P}=0.038)$. Postoperative Amsler testing was positive in five eyes in the peeling group and 13 eyes in the non-peeling group $(\mathrm{P}=0.024)$. Complications were similar in both groups; postoperatively, there were one and two cases of iatrogenic peripheral retinal break, three and two cases of macular hole, and one and one case of retinal detachment in the peeling and non-peeling groups, respectively.23G vitrectomy combined with brilliant blue-assisted ILM peeling resulted in better visual and
\end{abstract}

Correspondence to: Professor Zhipeng You, Department of Ophthalmology, The Second Affiliated Hospital of Nanchang University, 1 Minde Road, Nanchang, Jiangxi 330006, P.R. China E-mail: yzp74@sina.com

Key words: myopic foveoschisis, 23-gauge vitrectomy, internal limiting membrane peeling, brilliant blue anatomical effects compared with $23 \mathrm{G}$ vitrectomy alone in patients with MF.

\section{Introduction}

Myopic foveoschisis (MF) was first described in 1958 as a posterior retinal detachment without macular hole (1). It is a common complication in patients with myopia (2). It is characteristic of a macular intraretinal cleavage in myopic posterior staphyloma, and cannot be easily detected due to the poor contrast between the myopic retina and the choroid (3). However, hyporeflective splitting between the thin and faint reflective outer retina and the thicker, more reflective inner retina may be detected on an optical coherence tomography (OCT) scan (4), and its existence has been increasingly recognized with the arrival of OCT $(5,6)$. The estimated morbidity of MF ranges between 8 and 34\% in highly myopic eyes (7). The pathogenesis of MF is not clear. However, vitreous traction, decreased elasticity of the ILM, and a stretched retina due to staphyloma have been deemed to be possible causes (8). MF may be formed prior to macular retinal detachment and macular hole, and cause visual impairment, particularly when it affects the premacular structure $(9,10)$. Thus, in order to prevent the deterioration of the anatomy and function of the retina, and vision, surgical intervention is required for these patients (11). The effectiveness of $20 \mathrm{G}$ vitrectomy for MF has previously been demonstrated (12). The positive effect of vitrectomy also demonstrates that vitreous traction serves an important role in the development of MF. However, the surgical indications and the treatment time are not yet clear. Zhang et al (13) reported that MF and visual acuity (VA) deteriorated in 20 out of 29 eyes during a follow-up period of 31.2 months, and visual prognosis and surgical success rates have been poor following development of a macular hole in myopic eyes with posterior staphyloma (14).

Recently, there have been reports about minimally invasive vitrectomy surgery (MIVS) in treating MF (12). 23-gauge (23G) vitrectomy is one type of MIVS (15). The advantage of it is that the incision does not require suturing (16). The evidence also demonstrates that MIVS results in less inflammation, less discomfort, faster recovery of VA and, occasionally, a shorter surgery time $(12,17)$. The ILM can be more easily visualized with brilliant blue (BB) staining to aid peeling (15). 
Removal of traction by ILM peeling is a key component of numerous vitrectomy procedures (18). However, the evidence supporting the requirement to peel the ILM is inconsistent. Studies that indicate good outcomes following ILM peeling $(15,19,20)$ are countered by those that suggest that outcomes are improved without ILM peeling, or by methods that reduce the peeling area (21). However, the majority of these studies are case series that do not directly compare the two methods $(22,23)$, and those that do, suggest that peeling the ILM may be preferable (24). The present study hypothesized that $23 \mathrm{G}$ vitrectomy with BB-assisted ILM peeling in the eyes of patients with MF may provide a better outcome compared with $23 \mathrm{G}$ vitrectomy alone. Therefore, the aim of the current study was to compare the anatomical and visual outcomes of patients with MF treated by $23 \mathrm{G}$ vitrectomy with and without ILM peeling.

\section{Subjects and methods}

Study design. The current retrospective cohort study included data from patients treated with $23 \mathrm{G}$ vitrectomy for $\mathrm{MF}$ between March 2013 and August 2016 in the Department of Ophthalmology, The Second Affiliated Hospital of Nanchang University (Nanchang, China).

Patients. Patients were included in the study according to the following inclusion criteria: i) BCVA $>0.4$ (BCVA values were obtained using the $\log$ MAR test); ii) presented with metamorphopsia; iii) OCT examination revealed macular retinoschisis or macular retinoschisis with macular traction; and iv) refractive error $>-6.0$ diopters. The exclusion criteria were as follows: i) Eyes with an apparent macular hole; ii) eyes with retinal detachment; iii) eyes with glaucoma; and iv) eyes with choroidal neovascularization, macular degeneration or submacular hemorrhage.

The present study was performed in accordance with the tenets of the Declaration of Helsinki. The current study was approved by the Ethics Committee of The Second Affiliated Hospital of Nanchang University [approval no. (2012)095]. The requirement for written consent was waived by the Ethics Committee.

The patients were grouped to the peeling and non-peeling groups according to whether they received BB-assisted ILM peeling during the vitrectomy procedure or not. A total of 60 eyes from 60 patients were included in the study, and there were 30 eyes in each group.

Interventions. Patients in the peeling group underwent $23 \mathrm{G}$ pars plana vitrectomy (ppv) and ILM peeling with gas tamponade octafluoropropane (C3F8). Patients in the non-peeling group underwent $23 \mathrm{G}$ ppv with gas tamponade (C3F8). In the two groups, if patients had cataracts, phacoemulsification intraocular lens implantation (IOL) was performed prior to the posterior segment operation.

All operations were performed by the same experienced surgeon. The procedure for patients in the peeling and non-peeling groups was standard three-port $23 \mathrm{G}$ pars plana vitrectomy. To avoid the effects of postoperative cataract development, phacoemulsification with implantation of an intraocular lens was performed in the phakic eyes. The vitrectomy procedure consisted of core vitrectomy, creating a posterior vitreous detachment, peripheral vitrectomy and shaving of the basal vitreous body, the entire vitrectomy procedure was performed with the aid of Resight 700 non-contact wide-angle lenses (+128 Diopters; Resight; Carl Zeiss AG, Oberkochen, Germany). The residual posterior vitreous was identified using triamcinolone acetonide (Xudong Haipu Pharmaceuticals, Co., Ltd., Shanghai, China). The ILM was stained with $0.3 \mathrm{ml} \mathrm{BB}$ (Fluoron $\mathrm{GmbH}$, Ulm, Germany) in all patients of the peeling group, after entirely removing the posterior hyaloid membrane and epiretinal membrane. After $60 \mathrm{sec}$ treatment with BB, peeling of the ILM was performed by $23 \mathrm{G}$ ILM forceps and a non-contact wide-viewing lens (+60D) for an area of macular retinoschisis within the major vascular arcade and staphyloma. To avoid damage to the retina, the point of ILM peeling without inner retinoschisis was selected according to 3D analysis with visualization software on OCT (CIRRUS ${ }^{\mathrm{TM}}$ HD 4000-3716-OCT; Carl Zeiss Meditec, Inc., Dublin, CA). In 3D-OCT prior to vitrectomy, the outer retinoschisis (indicated by the white arrow) was observed in the macular area and inner retinoschisis (indicated by the white star) was observed in the temporal area of the maculae (Fig. 1A). The ILM was stained blue in the intraoperative camera under a +60D non-contact wide-viewing lens. The point of ILM peeling was selected in the nasal-inferior area of the maculae (indicated by white arrow; Fig. 1B). In the intraoperative camera under a +128D non-contact wide-viewing lens, no retinal break or hemorrhage was observed in the maculae following ILM peeling (indicated by white arrows; Fig. 1C).

After peeling ILM, air-gas (16\% C3F8) exchange was performed subsequent to liquid-air exchange. When leakage was found at the point of sclerotomy, the wound was sutured using 8-0 VICRYL ${ }^{\circledR}$ sutures (Johnson \& Johnson, New Brunswick, NJ, USA). Patients were asked to maintain a face-down position for 1 week.

Evaluations. All patients underwent ophthalmic examinations, including BCVA and CMT, at baseline and at the final visit. The examinations were undertaken using the $\log$ MAR test, Amsler test, indirect ophthalmoscopy, slit-lamp biomicroscopy, color fundus photography, OCT $(13,25)$ and axial length was measured with an optical biometer (IOL Master; Carl Zeiss AG). When OCT examination revealed no MF, the MF was considered completely resolved; when OCT examination revealed MF, and it was not considered to be as severe as the preoperative diagnosis, the MF was considered partly resolved. It was positive for Amsler's test when a vacancy or curve was found by looking at the white spot in the center of Amsler's grid.

Statistical analysis. All statistical analyses were performed with SPSS software (version 11.0; SPSS Inc., Chicago, IL, USA). Qualitative data are presented as a value and quantitative data are presented as the mean \pm standard deviation. Qualitative data were analyzed using a $\chi^{2}$ test or t-'s exact test. Continuous variables were analyzed using either the Wilcoxon signed-rank test or the Mann-Whitney test. $\mathrm{P}<0.05$ was considered to indicate a statistically significant difference.

\section{Results}

Patient demographics. A total of 30 eyes from 30 patients were included in each group (Table I). The baseline characteristics 
A

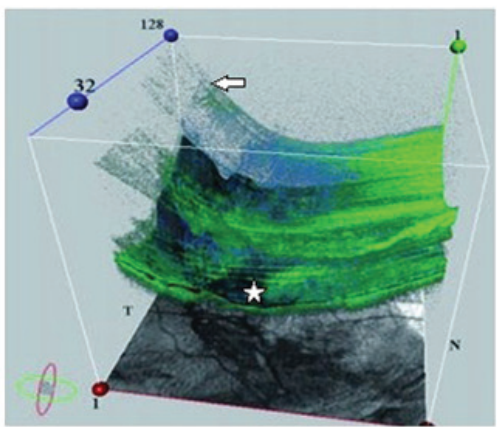

B

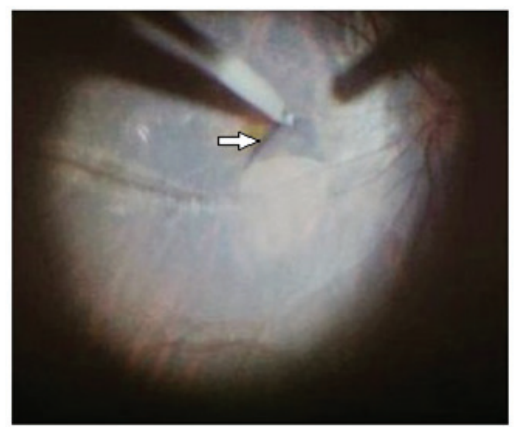

C

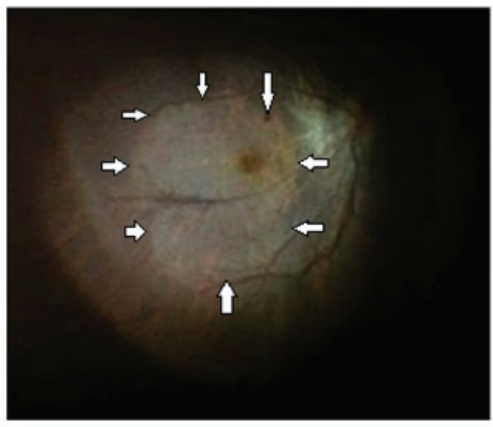

Figure 1. 3D-OCT-guided ILM peeling with brilliant blue staining. (A) 3D-OCT 1 week prior to vitrectomy. Outer retinoschisis (indicated by white arrow) was observed in the macular area, and inner retinoschisis (indicated by white star) was observed in the temporal area of the maculae. (B) Intraoperative camera under a +60D non-contact wide-viewing lens revealed that the ILM was stained blue. The arrow was used to indicate the blue staining of ILM. The point of ILM peeling was selected in the nasal-inferior area of the maculae (indicated by white arrow). (C) Intraoperative camera under a $+128 \mathrm{D}$ non-contact wide-viewing lens revealed no retinal break or hemorrhage in the maculae following ILM peeling (indicated by white arrows). 3D-OCT, three-dimensional optical coherence tomography; ILM, internal limiting membrane.

were similar between the two groups (all $\mathrm{P}>0.05)$. The mean age of the patients was $43.8 \pm 8.0$ years in the peeling group and $42.8 \pm 7.8$ years in the non-peeling group $(\mathrm{P}=0.774)$. The mean axial length was $28.3 \pm 1.2 \mathrm{~mm}$ in the peeling group compared with $28.5 \pm 1.2 \mathrm{~mm}$ in the non-peeling group $(\mathrm{P}=0.984)$. The mean follow-up duration post-surgery was $16.5 \pm 2.0$ months in the peeling group and $16.7 \pm 1.9$ months in the non-peeling group ( $\mathrm{P}=0.632)$. In the peeling group, $23(76.7 \%)$ of the 30 eyes were phakic prior to surgery and seven $(23.3 \%)$ eyes were pseudophakic. In the non-peeling group, 20 (66.7\%) eyes were phakic prior to surgery, and $10(33.3 \%)$ were pseudophakic. The mean preoperative BCVA ( $\log$ MAR) was $0.84 \pm 0.29$ in the peeling group and $0.81 \pm 0.30$ in the non-peeling group. The mean preoperative CMT was $458 \pm 62.2 \mu \mathrm{m}$ in the peeling group and $460 \pm 61.1 \mu \mathrm{m}$ in the non-peeling group. A total of 10 eyes had posterior staphyloma in the peeling group, and 11 eyes in the non-peeling group.

Visual and anatomical outcomes. Postoperative Visual acuity (logMAR) improved from the preoperative BCVA in the peeling and non-peeling group; however, the differences were not statistically significant (Table II). At the last follow-up, the visual acuity was improved in 24 out of 30 eyes (80\%) in the peeling group and 25 eyes $(83.3 \%)$ in the non-peeling group, remained unchanged in four $(13.3 \%)$ eyes in the peeling group and three $(10 \%)$ in the non-peeling group, and worsened in two eyes $(6.7 \%)$ in the peeling group and two eyes $(6.7 \%)$ in the non-peeling group. The overall difference was not significant $(\mathrm{P}=0.922)$. CMT decreased to $269.3 \pm 67.7 \mu \mathrm{m}$ in the peeling group and $294.4 \pm 60.5 \mu \mathrm{m}$ in the non-peeling group; this result was significantly different between the groups, with a greater improvement in the peeling group $(\mathrm{P}=0.015)$. In the peeling group, the foveoschisis on OCT completely resolved in all 30 eyes within 6 months, and this was only achieved in 26 eyes in the non-peeling group (Fig. 2). Significant differences were identified in the rate of CMT changes between the peeling group and the non-peeling group ( $\mathrm{P}=0.038$; Table II). Significant differences were also identified between the groups in the Amsler test, which was positive in five eyes in the peeling group and 13 eyes in the non-peeling group ( $\mathrm{P}=0.024$; Table II).
Postoperative complications. The complications were similar between the two groups (Table II). No serious complications, such as the iatrogenic macular hole, occurred during vitrectomy. Aniatrogenic peripheral retinal break developed in one eye from the peeling group and two eyes from the non-peeling group during vitrectomy, and the breaks were closed by intraoperative photocoagulation. A full-thickness macular hole developed in three eyes from the peeling group and two eyes from the non-peeling group postoperatively. A $0.3 \mathrm{cc}$ gas injection of $100 \%$ C3F8 was performed in combination with the maintenance of facedown position for 1 week, and the macular holes of the two eyes in the peeling group and two eyes in the non-peeling group were closed; however, the macular hole of one eye in the peeling group was not closed, and fluid-air exchange was performed followed by air-gas (10\% C3F8) exchange to finally close it. Retinal detachment developed in one eye of the peeling group and one eye of the non-peeling group post-vitrectomy due to a peripheral retinal break. The patients underwent the treatment with silicone oil injection and removal after 3 months; the retina reattached and the foveoschisis completely resolved. Due to leakage at sclerotomy, the wound was sutured in two eyes from the peeling group and three eyes from the non-peeling group.

\section{Discussion}

The aim of the present study was to investigate whether peeling the ILM during $23 \mathrm{G}$ vitrectomy was preferable to not peeling the ILM for patients with MF. The results demonstrated that BCVA improved in the peeling and non-peeling groups. CMT decreased more significantly in the peeling group compared with the non-peeling group. In the peeling group, MF completely resolved in all eyes of the peeling group, and this was only observed in $87 \%$ of eyes in the non-peeling group. In addition, the postoperative Amsler test was positive in just five eyes in the peeling group compared with 13 eyes in the non-peeling group, suggesting that any metamorphopsia had been resolved (26). Peeling the ILM led to no greater risk for patients, as complications were similar in the two groups. Therefore, it may be suggested that peeling the ILM may 
Table I. Baseline characteristics of the patients.

\begin{tabular}{|c|c|c|c|c|}
\hline Characteristics & $\begin{array}{c}\text { Peeling } \\
\text { group }(n=30)\end{array}$ & $\begin{array}{l}\text { Non-peeling } \\
\text { group }(n=30)\end{array}$ & P-value & Test \\
\hline Mean age $\pm \mathrm{SD}$, years (range) & $43.8 \pm 8.0$ & $42.8 \pm 7.8$ & 0.774 & Mann-Whitney test \\
\hline Gender (M/F) & $11 / 19$ & $12 / 18$ & 0.792 & $\chi^{2}$ test \\
\hline Mean $\mathrm{AX} \pm \mathrm{SD}, \mathrm{mm}$ (range) & $28.3 \pm 1.24$ & $28.5 \pm 1.21$ & 0.984 & Mann-Whitney test \\
\hline Preoperative IOP & $15.3 \pm 5.5$ & $15.0 \pm 5.2$ & 0.912 & Mann-Whitney test \\
\hline Duration of $\mathrm{MF} \pm \mathrm{SD}$, months (range) & $6 \pm 3.1$ & $6 \pm 3.3$ & 0.964 & Mann-Whitney test \\
\hline Preoperative BCVA in logMAR, mean \pm SD & $0.84 \pm 0.29$ & $0.81 \pm 0.30$ & 0.822 & Mann-Whitney test \\
\hline Preoperative CMT, mean \pm SD & $458 \pm 62.2$ & $460 \pm 61.1$ & 0.229 & Mann-Whitney test \\
\hline Amsler test positive, $\mathrm{n}$ & 21 & 22 & 0.781 & Mann-Whitney U test \\
\hline Preoperative lens status, $\mathrm{n}$ & & & 0.390 & $\chi^{2}$ test \\
\hline Phakic & 23 & 20 & & \\
\hline Pseudophakic & 7 & 10 & & \\
\hline Combined cataract surgery, $\mathrm{n}$ & 18 & 16 & 0.594 & Mann-Whitney U test \\
\hline Duration of Follow-up $\pm \mathrm{SD}$, months (range) & $16.5 \pm 2.0$ & $16.7 \pm 1.9$ & 0.632 & Mann-Whitney test \\
\hline $\mathrm{PS}, \mathrm{n}$ & 10 & 11 & 0.994 & Mann-Whitney U test \\
\hline
\end{tabular}

SD, standard deviation; M, male; F, female; AX, axial length; IOP, intraocular pressure; MF, myopic foveoschisis; BCVA, best-corrected visual acuity; CMT, central macular thickness; PS, posterior staphyloma; logMAR, Logarithm of the Minimum Angle of Resolution.

Table II. Postoperative outcomes and complications.

\begin{tabular}{|c|c|c|c|c|}
\hline & Peeling group & Non-peeling group & P-value & Statistics \\
\hline Postoperative BCVA in logMAR, mean \pm SD & $0.75 \pm 0.26$ & $0.72 \pm 0.25$ & 0.863 & Wilcoxon signed-rank test \\
\hline BCVA changes, $n$ & & & 0.922 & $\chi^{2}$ test \\
\hline Improved & 24 & 25 & & \\
\hline Unchanged & 4 & 3 & & \\
\hline Worsened & 2 & 2 & & \\
\hline Postoperative CMT, mean \pm SD & $269.3 \pm 67.7$ & $294.4 \pm 60.5$ & 0.015 & Mann-Whitney test \\
\hline CMT changes, $\mathrm{n}$ & & & 0.038 & Fisher's exact t-test \\
\hline Completely resolved & 30 & 26 & & \\
\hline Partly resolved & 0 & 4 & & \\
\hline Amslertest positive, $\mathrm{n}$ & 5 & 13 & 0.024 & $\chi^{2}$ test \\
\hline Complications & & & & $\chi^{2}$ test \\
\hline Iatrogenic peripheral retinal break & 1 & 2 & 0.554 & \\
\hline Macular hole & 3 & 2 & 0.643 & \\
\hline Retinal detachment & 1 & 1 & 1.0 & \\
\hline Wound suturation, $\mathrm{n}$ & 2 & 3 & 1.0 & $\chi^{2}$ test \\
\hline Gas tamponade (C3F8), n & 30 & 30 & 1.0 & $\chi^{2}$ test \\
\hline
\end{tabular}

SD, standard deviation; BCVA, best-corrected visual acuity; CMT, central macular; logMAR, Logarithm of the Minimum Angle of Resolution.

provide better outcomes compared with not peeling the ILM in 23-gauge vitrectomy.

Numerous surgical options for the treatment of foveoschisis have been reported (27). A number of studies have suggested the benefits of vitrectomy for the resolution of foveoschisis by removing vitreoretinal traction $(28,29)$. However, the evidence supporting the need to peel the ILM is inconsistent. Sayanagi et al (30) performed vitrectomy and
ILM peeling for persistent MF following primary surgery that did not include ILM peeling. Favorable anatomical and visual outcomes were achieved, indicating that ILM peeling may be crucial for the treatment of MF (31). A number of studies have reported that vitrectomy combined with ILM peeling results in better anatomical and visual effect compared with vitrectomy alone $(14,15,19,20,32)$. On the other hand, certain studies have demonstrated the effect of vitrectomy without 

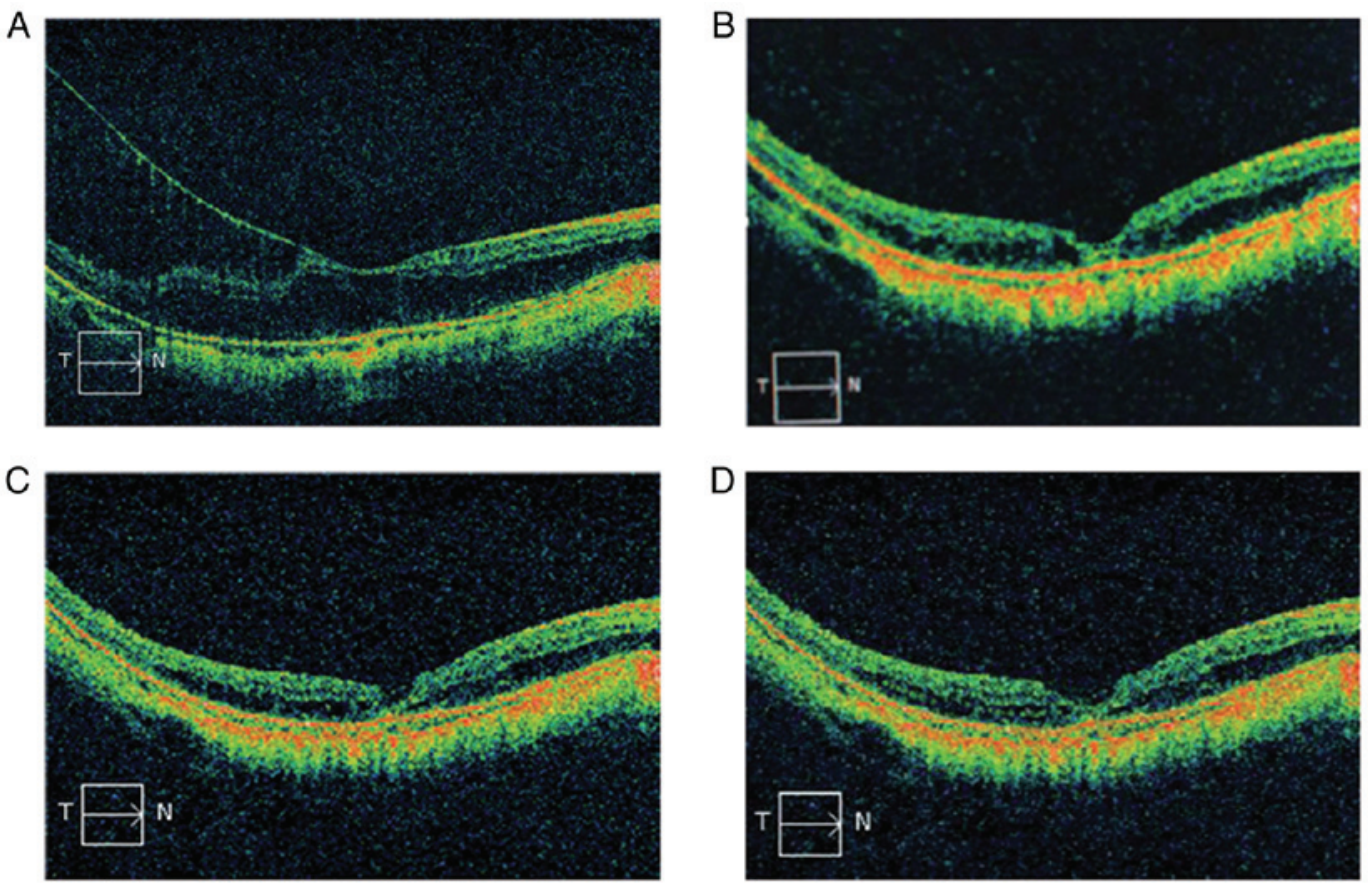

Figure 2. Representative OTC images for a case in the peeling group. Preoperative and postoperative OCT images are presented. The horizontal arrows indicate the direction of the OCT scans. (A) 1 week prior to vitrectomy. OCT demonstrated foveoschisis. Outer retinoschisis was observed in the fovea; inner retinoschisis was observed in the temporal area of the maculae. A columnar structure bridged the inner and outer retinal layers. VA was 1.40 logMAR. Central macular thickness was $500 \mu \mathrm{m}$. (B) Image captured 1 month after vitrectomy. The fovea was attached. VA was $1.0 \log$ MAR. CMT was $355 \mu \mathrm{m}$. (C) Image capture 3 months after vitrectomy. The retina remained attached. VA was $0.9 \log$ MAR. CMT was $300 \mu \mathrm{m}$. (D) Image captured 6 months after vitrectomy. The retina remained completely attached. VA was $0.4 \log$ MAR. CMT was $230 \mu \mathrm{m}$. OCT, optical coherence tomography; CMT, central macular thickness; VA, visual acuity; $\log$ MAR, Logarithm of the Minimum Angle of Resolution; T, temporal; N, nasal.

ILM peeling $(21,23,33)$. However, the majority of studies that have investigated ILM peeling are either case series or case reports (34), with very few studies comparing the two methods (35). The results of the present study suggested that ILM peeling may be the preferable method, and this result is consistent with the study which compared vitrectomy with and without ILM peeling by Taniuchi et al (24), which additionally demonstrated that tractional macular detachment developed more frequently in eyes without ILM peeling. To the best of our knowledge, the current study was the first to demonstrate improved rates of metamorphopsia resolution following ILM peeling, which is a major benefit in terms of the visual outcome. Although ILM peeling may be a risk factor for iatrogenic macular hole in high myopia (36), it ensures complete removal of all macular vitreous traction and reduces retinal stiffness to better match the posterior staphyloma (27). The thin and semitransparent ILM makes surgical visualization challenging in patients with ILM peeling. ILM peeling results in iatrogenic retinal injury involves macular hole irregularities of the nerve fiber layers and retinal microbleeds (37).

Chromovitrectomy has been developed as a way to improve the visibility of the ILM, in order to shorten the surgery duration and reduce iatrogenic retinal trauma (38). A variety of dyes, including trypan blue, indocyanine green (ICG) and BB, are used to dye the ILM (39). Though ICG was first used to peel the ILM in macular surgery, potential side effects have been reported (40). In the present study, BB, a relatively new type of dye, was used to dye the ILM. It selectively stains the ILM and has no toxicity (41-43). Furthermore, to avoid damage to the retina, the point area without inner retinoschisis was selected according to the $3 \mathrm{D}$ analysis with a $3 \mathrm{D}$ visualization software following OCT.

The present study has certain limitations. The sample size was quite small, and the patients were not randomized into groups due to the retrospective nature of the study. Therefore, there may have been certain bias in the patient selection. A larger study would be able to provide further evidence for these results and would allow the data to be analyzed according to different patient characteristics to evaluate whether different methods should be used in different patients. Vitrectomy combined with ILM peeling may solve the issue of vertical and tangent traction, although it cannot prevent the progress of posterior staphyloma, which remains an important factor in MF (27). Recently, a number of studies have shown that posterior scleral reinforcement surgery in the treatment of MF is effective; it may stabilize the eye axis and improve visual acuity $(44,45)$. Future studies may further investigate the therapeutic effect of vitrectomy combined with posterior scleral reinforcement on MF.

The current retrospective cohort study identified that $23 \mathrm{G}$ vitrectomy with BB-assisted ILM peeling resulted in more favorable visual and anatomical outcomes in patients with MF compared with not peeling the ILM.

\section{Acknowledgements}

The authors would like to thank the staff involved in the trial for their efforts in collecting and ensuring the accuracy and completeness of all data. 


\section{Funding}

The present study was supported by The Science and Technology Program of Jiangxi Provincial Health and Family Planning Commission of China (grant no. 20171073).

\section{Availability of data and materials}

All datasets used and/or analyzed during the present study are available from the corresponding author on reasonable request.

\section{Authors' contributions}

XM participated in the literature search, study design, surgery, data collection, data analysis and data interpretation, and wrote the manuscript. YC performed the data collection and analysis, and provided critical revisions. ZY conceived the study and participated in its design and coordination, and provided critical revisions. All authors read and approved the final manuscript.

\section{Ethics approval and consent to participate}

The current study was approved by the Ethics Committee of The Second Affiliated Hospital of Nanchang University [approval no. (2012)095].

\section{Patient consent for publication}

All patients provided written consent for publication.

\section{Competing interests}

The authors declare that they have no competing interests.

\section{References}

1. Phillips CI: Retinal detachment at the posterior pole. $\mathrm{Br}$ J Ophthalmol 42: 749-753, 1958.

2. Lim SJ, Kwon YH, Kim SH, You YS and Kwon OW: Vitrectomy and internal limiting membrane peeling without gas tamponade for myopic foveoschisis. Graefes Arch Clin Exp Ophthalmol 250: 1573-1577, 2012.

3. Ellabban AA, Tsujikawa A, Matsumoto A, Yamashiro K, Oishi A, Ooto S, Nakata I, Akagi-Kurashige Y, Miyake M, Elnahas HS, et al: Three-dimensional tomographic features of dome-shaped macula by swept-source optical coherence tomography. Am J Ophthalmol 155: 320-328.e2, 2013.

4. Zheng B, Chen Y, Chen Y, Zhao Z, Zhang Z, Zheng J, You Y, Wang $Q$ and Shen L: Vitrectomy and internal limiting membrane peeling with perfluoropropane tamponade or balanced saline solution for myopic foveoschisis. Retina 31: 692-701, 2011.

5. Wong TY, Ohno-Matsui K, Leveziel N, Holz FG, Lai TY, Yu HG, Lanzetta P, Chen Y and Tufail A: Myopic choroidal neovascularisation: Current concepts and update on clinical management. Br J Ophthalmol 99: 289-296, 2015.

6. Todorich B, Scott IU, Flynn HW Jr and Chang S: Macular retinoschisis associated with pathologic myopia. Retina 33: 678-683, 2013.

7. Meng B, Zhao L, Yin Y, Li H, Wang X, Yang X, You R, Wang J, Zhang $\mathrm{Y}$, Wang $\mathrm{H}$, et al: Internal limiting membrane peeling and gas tamponade for myopic foveoschisis: A systematic review and meta-analysis. BMC Ophthalmol 17: 166-176, 2017.

8. Faghihi H, Hajizadeh F and Riazi-Esfahani M: Optical coherence tomographic findings in highly myopic eyes. J Ophthalmic Vis Res 5: 110-121, 2010.
9. García-Ben A,BlancoMJ,PiñeiroA,MeraP,Rodriguez-AlvarezMX and Capeans C: Relationship between macular bending and foveoschisis in myopic patients. Optom Vis Sci 91: 497-506, 2014.

10. Mateo C, Gómez-Resa MV, Burés-Jelstrup A and Alkabes M: Surgical outcomes of macular buckling techniques for macular retinoschisis in highly myopic eyes. Saudi J Ophthalmol 27: 235-239, 2013.

11. García-Layana A, García-Arumí J, Ruiz-Moreno JM, AriasBarquet L, Cabrera-López F and Figueroa MS: A review of current management of vitreomacular traction and macular hole. J Ophthalmol 2015: 809640, 2015.

12. Gómez-Resa M, Burés-Jelstrup A and Mateo C: Myopic traction maculopathy. Dev Ophthalmol 54: 204-212, 2014.

13. Zhang T, Zhu Y, Jiang CH and Xu GZ: Long-term follow-up of vitrectomy in patients with pathologic myopic foveoschisis. Int J Ophthalmol 10: 277-284, 2017.

14. Kumagai K, Furukawa M, Ogino N and Larson E: Factors correlated with postoperative visual acuity after vitrectomy and internal limiting membrane peeling for myopic foveoschisis. Retina 30: 874-880, 2010.

15. Figueroa MS, Ruiz-Moreno JM, Gonzalez del Valle F, Govetto A, de la Vega C, Plascencia RN, Contreras I and Medina JL: Long-term outcomes of 23-gauge pars plana vitrectomy with internal limiting membrane peeling and gas tamponade for myopic traction maculopathy: A prospective study. Retina 35: 1836-1843, 2015.

16. Arevalo JF, Berrocal MH, Arias JD and Banaee T: Minimally invasive vitreoretinal surgery: Is sutureless vitrectomy the future of vitreoretinal surgery? J Ophthalmic Vis Res 6: 136-144, 2011.

17. Fujii GY, De Juan E Jr, Humayun MS, Chang TS, Pieramici DJ, Barnes A and Kent D: Initial experience using the transconjunctival sutureless vitrectomy system for vitreoretinal surgery. Ophthalmology 109: 1814-1820, 2002.

18. Ohta K, Sato A, Senda N and Fukui E: Comparisons of foveal thickness and slope after macular hole surgery with and without internal limiting membrane peeling. Clin Ophthalmol 12: 503-510, 2018.

19. VanderBeek BL and Johnson MW: The diversity of traction mechanisms in myopic traction maculopathy. Am J Ophthalmol 153: 93-102, 2012.

20. Hwang JU, Joe SG, Lee JY, Kim JG and Yoon YH: Microincision vitrectomy surgery for myopic foveoschisis. Br J Ophthalmol 97: 879-884, 2013.

21. Ho TC, Yang CM, Huang JS, Yang CH, Yeh PT, Chen TC, Ho A and Chen MS: Long-term outcome of foveolar internal limiting membrane nonpeeling for myopic traction maculopathy. Retina 34: 1833-1840, 2014.

22. Shimada N, Sugamoto Y, Ogawa M, Takase Hand Ohno-Matsui K: Fovea-sparing internal limiting membrane peeling for myopic traction maculopathy. Am J Ophthalmol 154: 693-701, 2012.

23. Qi Y, Duan AL, Meng X and Wang N: Vitrectomy without inner limiting membrane peeling for macular retinoschisis in highly myopic eyes. Retina 36: 953-956, 2016.

24. Taniuchi S, Hirakata A, Itoh Y, Hirota K and Inoue M: Vitrectomy with or without internal limiting membrane peeling for each stage of myopic traction maculopathy. Retina 33: 2018-2025, 2013.

25. Faes L, Bodmer NS, Bachmann LM, Thiel MA and Schmid MK: Diagnostic accuracy of the Amsler grid and the preferential hyperacuity perimetry in the screening of patients with age-related macular degeneration: Systematic review and meta-analysis. Eye (Lond) 28: 788-796, 2014.

26. Tranos P, Koukoula S, Charteris DG, Perganda G, Vakalis A, Asteriadis S, Georgalas I and Petrou P: The role of internal limiting membrane peeling in epiretinal membrane surgery: A randomised controlled trial. Br J Ophthalmol 101: 719-724, 2017.

27. Alkabes $\mathrm{M}$ and Mateo $\mathrm{C}$ : Macular buckle technique in myopic traction maculopathy: A 16-year review of the literature and a comparison with vitreous surgery. Graefes Arch Clin Exp Ophthalmol. May 256: 863-877, 2018.

28. Ikuno Y, Sayanagi K, Soga K, Oshima Y, Ohji M and Tano Y: Foveal anatomical status and surgical results in vitrectomy for myopic foveoschisis. Jpn J Ophthalmol 52: 269-276, 2008.

29. Elwan MM, Abd Elghafar AE, Hagras SM, Abou Samra WA and Saleh SM: Long-term outcome of internal limiting membrane peeling with and without foveal sparing in myopic foveoschisis. Eur J Ophthalmol 29: 69-74, 2019.

30. Sayanagi K,Ikuno Y and Tano Y: Reoperation for persistent myopic foveoschisis after primary vitrectomy. Am J Ophthalmol 141: 414-417, 2006. 
31. Seppey C and Wolfensberger TJ: Vitrectomy with fovea-sparing internal limiting membrane peeling for myopic foveoschisis. Klin Monbl Augenheilkd 234: 497-500, 2017.

32. Mii M, Matsuoka M, Matsuyama K, Otsu Y and Nishimura T: Favorable anatomic and visual outcomes with 25 -gauge vitrectomy for myopic foveoschisis. Clin Ophthalmol 8: 1837-1844, 2014.

33. Spaide RF and Fisher Y: Removal of adherent cortical vitreous plaques without removing the internal limiting membrane in the repair of macular detachments in highly myopic eyes. Retina 25: 290-295, 2005.

34. Ikuno Y, Sayanagi K, Ohji M, Kamei M, Gomi F, Harino S, Fujikado $\mathrm{T}$ and Tano Y: Vitrectomy and internal limiting membrane peeling for myopic foveoschisis. Am J Ophthalmol 137: 719-724, 2004

35. Liu HJ and Bi XJ: Vitrectomy combined with internal limiting membrane peeling for treating foveoschisis in high myopia. Int Eye Sci 10: 1871-1872, 2014.

36. Kwok AK, Lai TY and Yip WW: Vitrectomy and gas tamponade without internal limiting membrane peeling for myopic foveoschisis. Br J Ophthalmol 89: 1180-1183, 2005.

37. Sanisoglu H, Sevim MS, Aktas B, Sevim S and Nohutcu A Outcomes of 23-gauge pars plana vitrectomy and internal limiting membrane peeling with brilliant blue in macular hole. Clin Ophthalmol 5: 1177-1183, 2011.

38. Shukla D, Kalliath J, Neelakantan N, Naresh KB and Ramasamy K: A comparison of brilliant blue $\mathrm{G}$, trypan blue, and indocyanine green dyes to assist internal limiting membrane peeling during macular hole surgery. Retina 31: 2021-2025, 2011.

39. Farah ME, Maia M, Penha FM and Rodrigues EB: The use of vital dyes during vitreoretinal surgery-chromovitrectomy. Dev Ophthalmol 55: 365-375, 2016.
40. Yonekawa Y, Abbey AM, Shah AR, Thomas BJ and Capone A Jr: Endoilluminator phototoxic maculopathy associated with combined ICG-assisted epiretinal membrane and internal limiting membrane peeling. Clin Ophthalmol 8: 2501-2506, 2014.

41. Badaró E, Moraes-Filho M, Maia M, Penha FM, Novais EA, Souza-Lima RA, Hirai F, Meyer $\mathrm{CH}$, Farah ME and Rodrigues EB: Retinal biocompatibility of brilliant blue $\mathrm{g}$ with deuterated water for chromovitrectomy. J Ophthalmic Vis Res 9: 204-209, 2014

42. Januschowski K, Mueller S, Spitzer MS, Schramm C, Doycheva D, Bartz-Schmidt KU and Szurman P: Evaluating retinal toxicity of a new heavy intraocular dye, using a model of perfused and isolated retinal cultures of bovine and human origin. Graefes Arch Clin Exp Ophthalmol 250: 1013-1022, 2012.

43. Enaida H, Hisatomi T, Hata Y, Ueno A, Goto Y, Yamada T, Kubota $\mathrm{T}$ and Ishibashi T: Brilliant blue $\mathrm{G}$ selectively stains the internal limiting membrane/brilliant blue G-assisted membrane peeling. Retina 26: 631-636, 2006.

44. Zhu SQ, Zheng LY, Pan AP, Yu AY, WangQM and Xue AQ: The efficacy and safety of posterior scleral reinforcement using genipin cross-linked sclera for macular detachment and retinoschisis in highly myopic eyes. Br J Ophthalmol 5: 210-216, 2016.

45. Zhu Z, Ji X, Zhang J and Ke G: Posterior scleral reinforcement in the treatment of macular retinoschisis in highly myopic patients. Clin Exp Ophthalmol 37: 660-663, 2009.

(1) (-) This work is licensed under a Creative Commons Attribution-NonCommercial-NoDerivatives 4.0 International (CC BY-NC-ND 4.0) License. 